\title{
A Data-Driven Method for Predicting Deformation of Machined Parts Using Sparse Monitored Deformation Data
}

\author{
Zhiwei Zhao ${ }^{\mathrm{a}}$ Yingguang $\mathrm{Li}^{\mathrm{a}}$, 1 , Yee Mey Goh ${ }^{\mathrm{b}}$, Changqing Liu ${ }^{\mathrm{a}}$, Peter Kinnell ${ }^{\mathrm{b}}$ \\ ${ }^{a}$ National Key Laboratory of Science and Technology on Helicopter Transmission, \\ Nanjing University of Aeronautics and Astronautics, Nanjing, 21 0016, China \\ ${ }^{\mathrm{b}}$ Wolfson School of Mechanical, Electrical and Manufacturing Engineering, \\ Loughborough University, Loughborough, LE11 3TU, United Kingdom
}

\begin{abstract}
In the aircraft industry, where high precision geometric control is vital, unexpected component deformation, due to the release of internal residual stress, can limit geometric accuracy and presents process control challenges. Prediction of component deformation is necessary so that corrective control strategy can be defined. However, existing prediction methods, that are mainly based on the prediction or measurement of residual stress, are limited and accurate deformation prediction is still a research challenge. To address this issue, this paper presents a data-driven method for deformation prediction based on the use of in-process monitored deformation data. Deformation, which is caused by an unbalanced internal residual stress field, can be accurately monitored during the machining process via an instrumented fixture device. The state of the internal stress field within the part is first estimated by the using the part deformation data collected during machining process, and then, the deformation caused by a subsequent machining process is predicted. Deep learning is used to establish the estimating module and predicting module. The estimating module is used to infer the unobservable residual stress field as vectors by using sparse deformation data. The inferred vector is then used to predict the deformation in the predicting module. The proposed method provides an effective way to predict deformation during the machining of monolithic components, which is demonstrated experimentally.
\end{abstract}

Keywords. Machining deformation; Residual stress; Deformation prediction

\section{Introduction}

Machining deformation is one of the most crucial issue in manufacturing of aircraft structural parts, which causes geometric error and scrap parts. Machining deformation mainly results from the unbalanced residual stress distributed within the material[1]. Before machining, the internal residual stress distribution of the stock material is in equilibrium, however when some material is removed, equilibrium is lost, causing a new strain state within the material. Once fixtures are removed, the new strain state causes deformation of the part as it finds a new equilibrium state[2]. In this process, the initial

${ }^{1}$ Corresponding Author: Yingguang Li, Nanjing University of Aeronautics and Astronautics, Nanjing, 210016, China; E-mail: liyingguang@nuaa.edu.cn. 
residual stress is the main cause of the machining deformation, in comparison with cutting residual stress caused by heat or cutting forces[3].

To control this unwanted deformation the ability to predict deformation prior to machining is fundamental[4]. Existing research is mainly aimed at establishing the relationship between deformation and residual stress using numerical simulation methods[5] or analytical methods[6], in which the residual stress value is an inevitable initial condition. It means that the measurement of residual stress is necessary in deformation prediction. However, most of the measurement methods are off-line measurements, that are destructive, therefore representative samples, of material taken from a similar batch are used to represent the actual machined material. Due to the difference of distribution of residual stress, even within the same batch of material, there is of course the potential for differences between actual parts and samples[7]; as a result residual stress measurement for deformation prediction is a major unsolved research challenge.

A potential solution lies in the use of in-process monitoring technologies that are increasingly being used in the manufacturing industry to control machining process. The monitored in-process data such as clamping force[8] and cutting forces[9] can be used to monitor the development of local deformation, which in conjunction with appropriate deformation prediction models can be used for process control. In order to monitor machining deformation, the author's group has proposed a method using flexible fixtures[10]. This fixture system provides reference data for deformation prediction and control. Deformation prediction can be achieved by treating the problem as time-series prediction problem, and using the Kalman filtering[11] method or recurrent neural network[12] to predict machining deformation. However, as machining deformation complex due to the three dimensional nature of the geometry, and the associated residual stress field, time-series based prediction models can only deal with simple part with similar geometry in machining process.

In order to solve the problem, this paper proposes a machining deformation prediction method using monitored deformation data. In this method, the residual stress field is estimated first by first-pass machining deformation data, which is used to predict the initial stress state and the subsequent machining deformation. The prediction model is modeled by data-driven method, in which deep learning is used to learn the estimation and prediction ability.

\section{Methodology}

Machining deformation is mainly caused by initial residual stress. In a machining process, the part is fixed on the workbench, in which the geometry is constrained by fixtures. It means that, whilst the part is fixed, there is no strain produced in the part unmachined areas, and the residual stress remains unchanged in those areas. With this assumption, the initial residual stress field can be deemed as a stable space field. The machining deformation is caused by the stable field and the changeable variable, geometry.

The relationship could be expressed as,

$$
d_{i, j}=f\left(I R S, G_{j}, C_{i}\right)
$$


Where IRS is the Initial Residual Stress field within the material, $C_{i}$ is the coordinates of the $i$-th monitored deformation position, $G_{j}$ is the geometry in after $j$-th machining step, and each machining step removes a certain area of material, $d_{i, j}$ is the monitored deformation in $j$-th geometry at coordinates $i$.

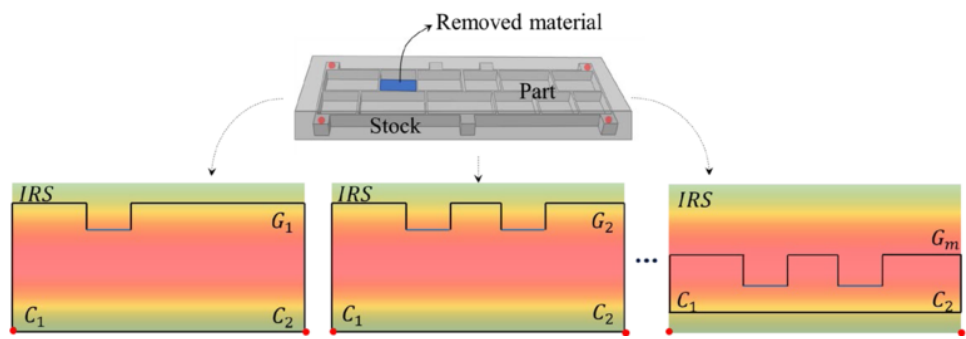

Figure 1 The diagram of machining deformation

The IRS could be estimated by monitored deformation data,

$$
I R S=g\left(d_{1 \ldots n, 1}, G_{1}, C_{1 \ldots n}, d_{1 \ldots n, 2}, G_{2}, C_{1 \ldots n}, d_{1 \ldots n, m}, G_{m}, C_{1 \ldots n,}\right)
$$

Where $\mathrm{m}$ is the number of machining steps, $\mathrm{n}$ is number of the deformation monitoring points. The $\left(d_{1 \ldots n, j}, G_{j}, C_{1 \ldots n}\right)$ could be seen as a representation of state of $j$ th machining process.

Each deformation data is generated by IRS, so it could be deemed as a glimpse of the IRS, as shown in Figure 1. The whole IRS could be estimated by some sparse deformation data.

$$
I R S=g\left(d_{1 \ldots n, p}, G_{p}, C_{1 \ldots n}, \ldots d_{1 \ldots n, q}, G_{q}, C_{1 \ldots n}\right) \quad 0<p<q<m
$$

When the estimated IRS is obtained, it could be used to predict the deformation further based on Eq.(1). In this process, the IRS is a latent variable, which is not the real value but a representation of residual stress filed.

\section{Implementation}

The above method could be concluded that using part of the known information related to deformation $\left(G_{c}, C_{c}, d_{c}\right)$ to infer the latent variable $I R S$, and $I R S$ will be used to predict the target deformation $\left(d_{T}\right)$ with the known related variable $\left(G_{T}, C_{T}\right)$. The variable inference graph is shown in Figure 2.

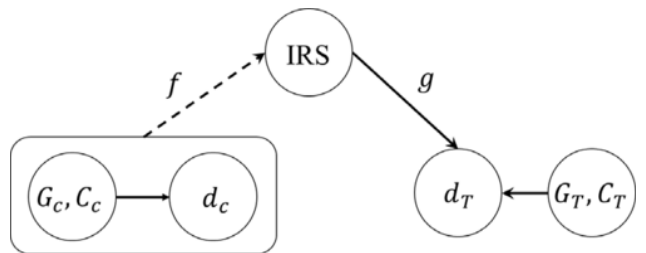

Figure 2 Relationship of machining deformation related variables 
The whole machining deformation prediction model has two modules, estimation module $g$ and prediction module $f$. It is clear that the functions $f$ and $g$ are difficult to obtain by analytical method. In order to form the functions, deep learning is used to model them.

\subsection{Framework of estimation module}

As for each machining step, there are a certain number of monitored deformation forces monitored by fixture devices. So, the full set of deformation forces, for a machining step with uniform geometry, are combined as a tensor and this is used to extract useful information. The extraction process is realised by neural network, included convolutional neural network and full-connection neural network. And collecting the information extracted from known machining steps to obtain the IRS, which is represented by vector. The structure of the estimation module is shown in Figure 3.

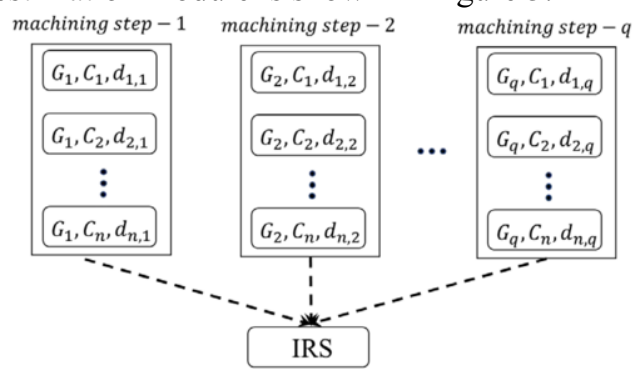

Figure 3 Structure of the estimation module

\subsection{Framework of prediction module}

When obtaining the IRS vector, the machining deformation of specific position for the follow-up machining step could be predicted using IRS vector, geometry of machining step and monitoring coordinate of deformation, as shown in Figure 4. This module is implemented by fully-connected neural network and convolutional neural network, in which the convolutional neural network is used to handle high-dimensional geometry tensor.

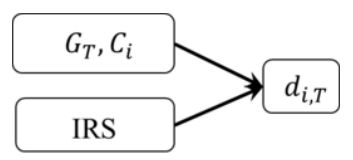

Figure 4 Structure of the prediction module

\subsection{Training option}

The Loss function of the network is based on mean square.

$$
\text { Loss }=\frac{1}{n * m} \sum_{i=1}^{n * m}\left(d_{\text {real }_{i}}-d_{\text {predicted }_{i}}\right)^{2}
$$

The training method is mini-batch stochastic gradient descent with the batch-size of 8 . And the learning rate starts is $1 \times 10^{-5}$. The absolute value error is used to evaluate the model performance. 


\section{Experiment}

The prediction model is trained on a total number of 350 parts samples with more than 20000 machining steps, and tested on a set with a number of 100 parts. All this samples are generated in simulation environment. In order to make full use of the limited data, a random sample training method is used. Some deformation data could be randomly selected from the whole machining deformation data of a single part to be deemed as known data and the remaining data is used as target data needed to be predicted. It means that a series of deformation data of a part could be used for many times in training process by random partition.

(a)

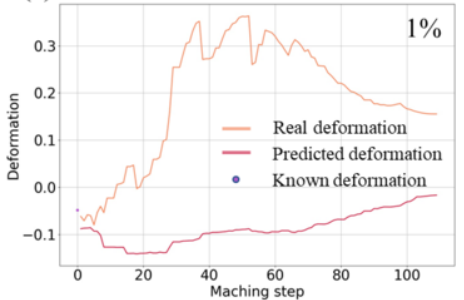

(c)

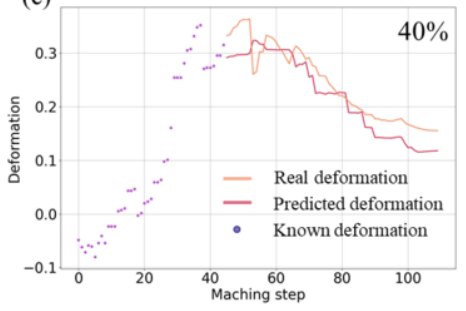

(b)

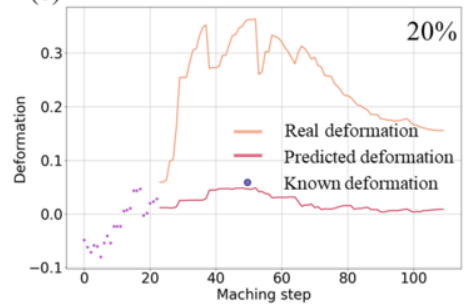

(d)

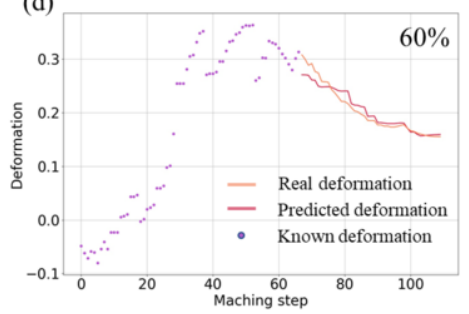

Figure 5 Result of a deformation prediction sample

A deformation prediction sample is illustrated to show the prediction performance in Figure 5. The prediction performances with $1 \%, 20 \%, 40 \%$ and $60 \%$ of the known deformation are shown in Figure 5(a), (b), (c), and (d) respectively. It can be seen that, with the number of increasing known deformation data increasing, the performance of the prediction model becomes better.

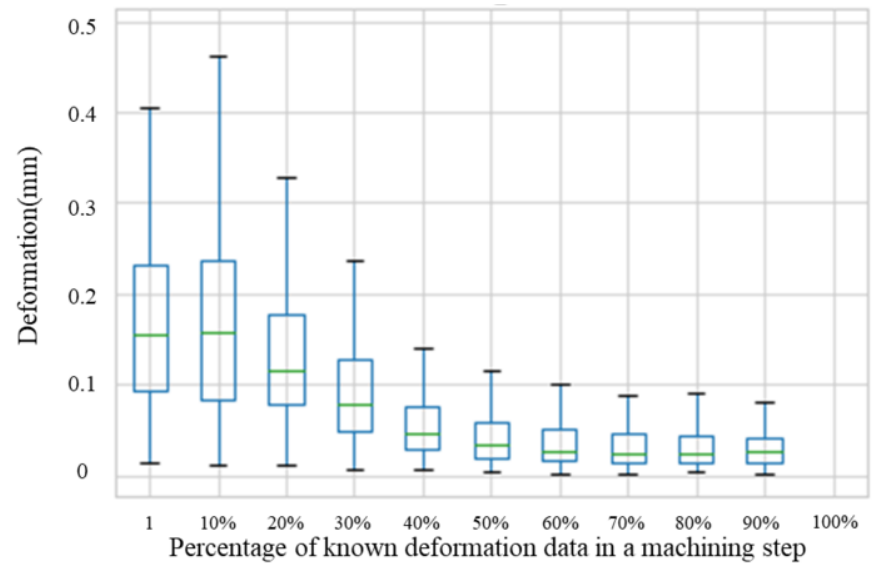

Figure 6 Mean errors of deformation prediction model using different amount of deformation data 
In order to validate the performance of the prediction model, the mean prediction errors with different amount of known deformation data are showed in Figure.6. It can be seen from the figure that, when the percentage of the known data is bigger than $60 \%$, and the center of the error is around $0.03 \mathrm{~mm}$, the max prediction error is nearly $0.1 \mathrm{~mm}$.

\section{Conclusion and further work}

This paper proposed a deformation prediction method based on latent inference and deep learning using sparse monitored deformation data. Different from deformation prediction methods based on time-series problems using a recurrent neural network, this method translates the time series property of the deformation prediction problem to a dis-ordered spatial field estimation problem, which allows the extraction of more useful information; an improve the overall residual stress field and deformation prediction performance. The validity of the model is verified on the simulation data set. On-going and further work of the authors is to integrate a Bayesian framework with proposed method to model the uncertainty of the prediction model and to provide a more reliable reference for the deformation control in actual machining environment.

\section{References}

[1] Barcenas L, Ledesma-Orozco E, Van-der-Veen S, Reveles-Arredondo F, Rodríguez-Sánchez E A. An Optimization of Part Distortion for a Structural Aircraft Wing Rib: An Industrial Workflow Approach. CIRP Journal of Manufacturing Science and Technology, 2020, 28(2019): 15-23.

[2] Zhang Z, Luo M, Tang K, Zhang D. A New In-Processes Active Control Method for Reducing the Residual Stresses Induced Deformation of Thin-Walled Parts. Journal of Manufacturing Processes, 2020, 59(9): 316-325.

[3] Chantzis D, Van-der-Veen S, Zettler J, Sim W M. An Industrial Workflow to Minimise Part Distortion for Machining of Large Monolithic Components in Aerospace Industry. Procedia CIRP, 2013, 8: 281286.

[4] Nervi S, Szabó B A, Young K A. Prediction of Distortion of Airframe Components Made from Aluminum Plates. AIAA Journal, 2009, 47(7): 1635-1641.

[5] Huang X, Sun J, Li J. Finite Element Simulation and Experimental Investigation on the Residual Stress-Related Monolithic Component Deformation. International Journal of Advanced Manufacturing Technology, 2015, 77(5-8): 1035-1041.

[6] Wu Q, Li D P, Ren L, Mo S. Detecting Milling Deformation in 7075 Aluminum Alloy Thin-Walled Plates Using Finite Difference Method. International Journal of Advanced Manufacturing Technology, 2016, 85(5-8): 1291-1302.

[7] Schajer G S. Practical Residual Stress Measurement Methods[M]. Practical Residual Stress Measurement Methods. .

[8] Gonzalo O, Seara J M, Guruceta E, Izpizua A, Esparta M, Zamakona I, Uterga N, Aranburu A, Thoelen J. A Method to Minimize the Workpiece Deformation Using a Concept of Intelligent Fixture. Robotics and Computer-Integrated Manufacturing, 2017, 48(4): 209-218.

[9] Möhring H C, Litwinski K M, Gümmer O. Process Monitoring with Sensory Machine Tool Components. CIRP Annals - Manufacturing Technology, 2010, 59(1): 383-386.

[10] Li Y, Liu C, Hao X, Gao J X, Maropoulos P G. Responsive Fixture Design Using Dynamic Product Inspection and Monitoring Technologies for the Precision Machining of Large-Scale Aerospace Parts. CIRP Annals - Manufacturing Technology, 2015, 64(1): 173-176.

[11] Hao X, Li Y, Li M, Liu C. A Part Deformation Control Method via Active Pre-Deformation Based on Online Monitoring Data. International Journal of Advanced Manufacturing Technology, 2019, 104(58): 2681-2692.

[12] Zhao Z, Li Y, Liu C, Gao J. On-Line Part Deformation Prediction Based on Deep Learning. Journal of Intelligent Manufacturing, 2020, 31: 561-574. 